\title{
Bordered Conjugates of Words over Large Alphabets
}

\author{
Tero Harju \\ University of Turku \\ harju@utu.fi
}

\author{
Dirk Nowotka \\ Universität Stuttgart \\ nowotka@fmi.uni-stuttgart.de
}

Submitted: Oct 23, 2008; Accepted: Nov 14, 2008; Published: Nov 24, 2008

Mathematics Subject Classification: 68R15

\begin{abstract}
The border correlation function attaches to every word $w$ a binary word $\beta(w)$ of the same length where the $i$ th letter tells whether the $i$ th conjugate $w^{\prime}=v u$ of $w=$ $u v$ is bordered or not. Let $[u]$ denote the set of conjugates of the word $w$. We show that for a 3-letter alphabet $A$, the set of $\beta$-images equals $\beta\left(A^{n}\right)=B^{*} \backslash\left(\left[a b^{n-1}\right] \cup D\right)$ where $D=\left\{a^{n}\right\}$ if $n \in\{5,7,9,10,14,17\}$, and otherwise $D=\emptyset$. Hence the number of $\beta$-images is $B_{3}^{n}=2^{n}-n-m$, where $m=1$ if $n \in\{5,7,9,10,14,17\}$ and $m=0$ otherwise.
\end{abstract}

Keywords: combinatorics on words, border correlation, binary words, square-free, cyclically square-free, Currie set,

\section{Introduction}

The border correlation function of a word was introduced by the present authors in [4], where the binary case was considered in detail. In this paper we consider the case for alphabets of size $s \geq 3$. The border correlation function is related to the auto-correlation function of Guibas and Odlyzko [3], as well as to the border-array function of Moore, Smyth and Miller [7]. Border correlation of partial words have been recently considered by Blanchet-Sadri et al. [1].

A word $w \in A^{*}$ is said to be bordered (or self-correlated [8]), if there exists a nonempty word $v$, with $v \neq w$, such that $w=u_{1} v=v u_{2}$ for some words $u_{1}, u_{2}$. In this case $v$ is a border of $w$. A word that has a border is called bordered; otherwise it is unbordered.

Let $\sigma: A^{*} \rightarrow A^{*}$ be the (cyclic) shift function, where $\sigma(x w)=w x$ for all $w \in A^{*}$ and $x \in A$, and $\sigma(\varepsilon)=\varepsilon$ for the empty word $\varepsilon$. Let $B=\{a, b\}$ be a special binary alphabet. The border correlation function $\beta: A^{*} \rightarrow B^{*}$ is defined as follows. For the empty word, let $\beta(\varepsilon)=\varepsilon$. For a word $w \in A^{*}$ of length $n$, let $\beta(w)=c_{0} c_{1} \ldots c_{n-1} \in B^{*}$ be the binary 
word of the same length such that

$$
c_{i}= \begin{cases}a & \text { if } \sigma^{i}(w) \text { is unbordered } \\ b & \text { if } \sigma^{i}(w) \text { is bordered }\end{cases}
$$

Example 1. (1) Assume the word $w$ is not primitive, i.e., $w=u^{k}(=u u \ldots u)$, for some power $k \geq 2$. Then all words $\sigma^{i}(w)$ are bordered, and thus $\beta(w)=b^{n}$, where $n$ is the length of $w$.

(2) Consider the alphabet $A=\{a, b, c\}$, and let $w=b a c a b a \in A^{*}$. Then

\begin{tabular}{c|c|c||c|c|c}
$i$ & $\sigma^{i}(w)$ & border & $i$ & $\sigma^{i}(w)$ & border \\
\hline 0 & bacaba & $b a$ & 3 & $a b a b a c$ & - \\
1 & acabab & - & 4 & babaca & - \\
2 & cababa & - & 5 & abacab & $a b$
\end{tabular}

and hence $\beta(w)=$ baaaab. Note that a border need not be unique.

For an alphabet $A$, let $A^{*}$ denote the monoid of all finite words over $A$ including the empty word $\varepsilon$. Also, let $A^{n}$ denote the set of words $w \in A^{*}$ of length $n$. In the binary case, where we can choose $A=B(=\{a, b\})$, it was shown in [4] that the image $\beta(w)$ of $w \in B^{*}$ does not have two consecutive $a$ 's except for some trivial cases. Hence, if $\sigma^{i}(w)$ is unbordered, then $\sigma^{i+1}(w)$ is necessarily bordered. Also, in the binary case, there are other 'exceptions', e.g., for no binary word $w$, it is the case that $\beta(w)=a b a b a b b a b a b b$. It is an open problem to characterize the set of the images $\beta(w)$ for $w \in B^{*}$.

The words $x y$ and $y x$ are called conjugates of each other. We denote by $[w]$ the set of all conjugates of the word $w$. Note that if $u$ and $v$ are conjugates then $v=\sigma^{i}(u)$ for some $i$, and hence, for all words $w$,

$$
\beta([w])=[\beta(w)] .
$$

Let $\beta\left(A^{n}\right)=\left\{\beta(w) \mid w \in A^{n}\right\}$ be the set of the $\beta$-images of the words of length $n$, and denote by $B_{k}^{n}$ the cardinality of $\beta\left(A^{n}\right)$ where $A$ is a $k$-letter alphabet. In the present paper we prove the following result, where

$$
\mathbf{C}=\{5,7,9,10,14,17\}
$$

is the Currie set of integers.

Theorem 1. Let $A$ be an alphabet of three letters, and let $n \geq 2$. Then

$$
\beta\left(A^{n}\right)= \begin{cases}B^{*} \backslash\left[a b^{n-1}\right] & \text { if } n \notin \mathbf{C}, \\ B^{*} \backslash\left(\left[a b^{n-1}\right] \cup\left\{a^{n}\right\}\right) & \text { if } n \in \mathbf{C} .\end{cases}
$$

In particular, $B_{3}^{n}=2^{n}-n-m$, where $m=1$ if $n \in \mathbf{C}$ and $m=0$ otherwise. 
We end this section with some definitions and notation needed in the rest of the paper. We refer to Lothaire's book [6] for more basic and general definitions of combinatorics on words.

We denote the length of a word $w$ by $|w|$. A word $u$ is a factor of a word $w \in A^{*}$, if $w=w_{1} u w_{2}$ for some words $w_{1} \in A^{*}$ and $w_{2} \in A^{*}$. A word $w \in A^{*}$ is said to be square-free, if it does not have a factor of the form $v v$ where $v \in A^{*}$ is nonempty. Moreover, $w$ is cyclically square-free, if all its conjugates are square-free.

\section{The proof}

This section let $A=\{a, b, c\}$ be a ternary alphabet. Let $T$ denote the Thue word obtained by iterating the substitution $\varphi:\{a, b, c\}^{*} \rightarrow\{a, b, c\}^{*}$ determined by $\varphi(a)=a b c, \varphi(b)=a c$ and $\varphi(c)=b$. Therefore $T$ is the infinite word starting with

$$
T=a b c a c b a b c b a c a b c a c b a c a b c b a \ldots
$$

As was shown by Thue $[9,10]$ (see also Lothaire [5]), the word $T$ is square-free, i.e., it does not contain any nonempty factors of the form $v v$.

Recall that $[w]$ denotes the conjugacy class of the word $w$. By the next lemma, each primitive word has at least two unbordered conjugates.

Lemma 1. For all $n \geq 2,\left[a b^{n-1}\right] \cap \beta\left(A^{n}\right)=\emptyset$.

Proof. Assume $a$ occurs in $\beta(w)$ for a word $w$ with $|w| \geq 2$. Hence $w$ is primitive. A conjugate $v$ of $w$ is a Lyndon word if it is minimal in $[w]$ with respect to some lexicographic order of $A^{*}$. It is well known (see, e.g., Lothaire [6]), that each primitive word $w$ has a unique Lyndon conjugate with respect to a given order and that each Lyndon word is unbordered. Hence, there exists at least two Lyndon words in $[w]$ for a given order of $A$ and its inverse order, respectively. These two words imply that $a$ occurs at least twice in $\beta(w)$.

The following result is due to Currie [2].

Theorem 2 (Currie). There exists a cyclically square-free word $w \in A^{n}$, if and only if $n \notin \mathbf{C}=\{5,7,9,10,14,17\}$.

A square $v v$ is called simple if $v \in a^{*}$ with $v \neq \varepsilon$. Let $w_{(i)}$ denote the $i$-th letter of $w$.

Lemma 2. Let $w$ be a square-free word. Then $w^{\prime}=w_{(1)}^{k_{1}} w_{(2)}^{k_{2}} \cdots w_{(n)}^{k_{n}}$ contains only simple squares for all $1 \leq i \leq n$ and $k_{i} \geq 1$.

Proof. Suppose on the contrary that $w^{\prime}$ contains a nonsimple square $v v$, say

$$
\begin{aligned}
v & =b_{i+1}^{p_{i+1}} b_{i+2}^{p_{i+2}} \cdots b_{i+j-1}^{p_{i+j-1}} b_{i+j}^{p_{i+j}} \\
& =b_{i+j+1}^{p_{i+j+1}} b_{i+j+2}^{p_{i+j+2}} \cdots b_{i+2 j-1}^{p_{i+2 j-1}} b_{i+2 j}^{p_{i+2 j}}
\end{aligned}
$$


with $0 \leq i \leq n-2 j$ and $p_{i+1} \leq k_{i+1}$ and $p_{i+\ell}=k_{i+\ell}=k_{i+j+\ell-1}$, for all $2 \leq \ell<j$, and $p_{i+j}+p_{i+j+1}=k_{i+j}$ and $p_{i+j} \leq k_{i+2 j-1}$ and $b_{i+1}=b_{i+j}=b_{i+2 j}=w_{(i+j)}=w_{(i+2 j-1)}$ and $b_{i+\ell}=b_{i+j+\ell}=w_{(i+\ell)}=w_{(i+j+\ell-1)}$, for all $1 \leq \ell<j$.

Observe that we obtain a square $\left(b_{i+1} b_{i+2} \cdots b_{i+j-1}\right)^{2}$ from $v v$ when all powers in $v v$ are reduced to 1 and the last letter is deleted. But now, we have that $b_{i+1} b_{i+2} \cdots b_{i+j-1}=$ $w_{(i+1)} w_{(i+2)} \cdots w_{(i+j-1)}=w_{(i+j)} w_{(i+j+1)} \cdots w_{(i+2 j-2)}$ implies a square in $w$; a contradiction.

Lemma 3. Let $w$ be a cyclically square-free word of length $n \geq 2$. Then for each nonempty $u \in\{a, b\}^{*}$ that has exactly $n$ occurrences of a, there exists a word $w^{\prime}$ such that $\beta\left(w^{\prime}\right)=u$.

Proof. By (1), we can assume without loss of generality that $u$ begins with the letter $a$. Let $u=a b^{k_{1}} a b^{k_{2}} \cdots a b^{k_{n}}$ where $k_{i} \geq 0$, for all $1 \leq i \leq n$. By Lemma 2, $w^{\prime}=w_{(1)}^{k_{1}+1} w_{(2)}^{k_{2}+1} \cdots w_{(n)}^{k_{n}+1}$ and all its conjugates contain only simple squares. That is, if a conjugate $w_{(i)}^{k_{i}+1} w_{(i+1)}^{k_{i+1}+1} \cdots w_{(n)}^{k_{n}+1} w_{(1)}^{k_{1}+1} \cdots w_{(i-1)}^{k_{i-1}+1}$ of $w^{\prime}$ that starts and ends in different letters is bordered then $w_{(i)} w_{(i+1)} \cdots w_{(n)} w_{(1)} \cdots w_{(i-1)}$ is bordered contradicting the fact that $w$ is cyclically square-free. This means that every conjugate of $w^{\prime}$ that starts and ends in a different letter is unbordered and all other conjugates are, of course, bordered by a border of length one. Hence, we have $\beta\left(w^{\prime}\right)=u$ which completes the proof.

Lemma 4. Let $n \in \mathbf{C}$. Then $u=a b^{k_{1}} a b^{k_{2}} \cdots a b^{k_{n}} \in \beta\left(A^{*}\right)$ whenever $u \notin a^{*}$.

Proof. Consider the following six words with lengths in $\mathbf{C}$ which have a unique border $v$ of length two or three (the borders are underlined):

\section{5: $\underline{a b} \underline{a b}$ \\ 7: $\underline{a b c} \underline{a b c} \underline{a b}$ \\ 9: $\underline{a b c a c b c \underline{a b}}$ \\ 10: $\underline{a b c a c b a c \underline{a b}}$ \\ 14: $\underline{a b c b a c a b a c b a b c}$ \\ 17: $\underline{a b c a b a c b c a b c b a c \underline{a b}}$}

It is straightforward to check that for every word $w$ in the list, each $x \in[w]$ with $x \neq w$ is unbordered, i.e., there exists only one bordered word $w$ in the conjugacy class $[w]$ and $w$ has a unique border. This also implies that these words are square-free.

Let

$$
u=a b^{k_{1}} a b^{k_{2}} \cdots a b^{k_{n}}
$$

as in the statement of the lemma.

We proceed by case distinction on $|v|$ to show that for every $n$ there exists a word $w^{\prime}$ such that $\beta\left(w^{\prime}\right)=u$ except if $k_{1}=k_{2}=\cdots=k_{n}$ for $n$ equal to $5,7,9,14$, or 17 , and $k_{1}=k_{3}=k_{5}=k_{7}=k_{9}$ and $k_{2}=k_{4}=k_{6}=k_{8}=k_{10}$ for $n=10$. The exceptional cases are handled at the end of the proof. 
Let $w \in A^{*}$ be any square-free word having a unique border $v$ such that each word in $[w] \backslash\{w\}$ is unbordered. Write $w=w_{(1)} w_{(2)} \ldots w_{(n)}$, where again $w_{(i)}$ denotes the $i$ th letter of $w$.

Suppose first that $|v|=3$ as in the case for 7 and 14. We can assume that $v=a b c$ (possibly by renaming the letters); otherwise $v$ would not be a unique border. Hence $w_{(1)} w_{(2)} w_{(3)}=a b c=w_{(n-2)} w_{(n-1)} w_{(n)}$. Consider $w^{\prime}=w_{(1)}^{k_{1}+1} w_{(2)}^{k_{2}+1} \cdots w_{(n)}^{k_{n}+1}$. Since exactly one conjugate of $w$ is bordered, the number of the letter $a$ in the $\beta$-image equals $n$, if $w^{\prime}$ is unbordered. Now, $w^{\prime}$ is unbordered if $k_{2} \neq k_{n-1}$, and in this case $\beta\left(w^{\prime}\right)=u$. Note that, by (1), it is enough to show that $\beta\left(w^{\prime}\right)=u^{\prime}$ for any conjugate $u^{\prime}$ of $u$. In particular, we are done if the powers $k_{i}$ can be cycled so that, for some $j$, the word $w^{\prime \prime}=w_{(1)}^{k_{1}^{\prime}+1} w_{(2)}^{k_{2}^{\prime}+1} \cdots w_{(n)}^{k_{n}^{\prime}+1}$, where $k_{i}^{\prime}=k_{i+j \bmod n}$, is unbordered. It follows that, for the border length 3 , the only cases left in $n \in \mathbf{C}$ are when $k_{1}=k_{2}=\cdots=k_{n}$. (Note that the case $n=9$, where $n$ is divisible by 3 , is treated below.)

Suppose then that $|v|=2$ as in the case for 5, 9, 10, and 17. We can assume that $v=a b$ (possibly after renaming of the letters), i.e., $w_{(1)} w_{(2)}=a b=w_{(n-1)} w_{(n)}$. Consider $w^{\prime}=w_{(1)}^{k_{1}+1} w_{(2)}^{k_{2}+1} \cdots w_{(n)}^{k_{n}+1}$. We recall that $w$ is the unique bordered word in its conjugacy class. Now, $w^{\prime}$ is unbordered if $k_{1}>k_{n-1}$ or $k_{2}<k_{n}$. Analogously to the above case with $|v|=3$ we can consider shifts of the indices modulo $n$. We conclude that $w^{\prime}$ is bordered for all possible shifts of $k_{1}, k_{2}, \ldots, k_{n}$ only if $k_{1}=k_{2}=\cdots=k_{n}$ or $n$ is even; a case that is avoided for $|v|=2$ except for $n=10$. If $n=10$ then we are left with the case where $k_{1}=k_{3}=\cdots=k_{9}$ and $k_{2}=k_{4}=\cdots=k_{10}$, where possibly $k_{1}=k_{2}$.

It remains to be shown that $u$ is a $\beta$-image if $k_{1}=k_{2}=\cdots=k_{n}$ or $k_{1}=k_{3}=\cdots=k_{9}$ and $k_{2}=k_{4}=\cdots=k_{n}$, if $n=10$, with $k_{i} \geq 1$ for all $1 \leq i \leq n$. Let $t=k_{1}+1$ and $s=k_{2}+1$. The following list gives a word for every $n \in \mathbf{C}$ such that the $\beta$-image is $\left(a b^{t-1}\right)^{n}$ or $\left(a b^{t-1} a b^{s-1}\right)^{5}$ in the case $n=10$.

$$
\begin{aligned}
& \text { 5: } a^{t} b^{t} c^{t} a^{t} b c^{t-1} \\
& \text { 7: } a^{t} b^{t} c^{t} b^{t} a^{t} b^{t} c b^{t-1} \\
& \text { 9: } a^{t} c^{t} b^{t} a^{t} b^{t} c^{t} b^{t} a^{t} c b^{t-1} \\
& \text { 10: } c^{t} b^{s} a^{t} c^{s} a^{t} b^{s} c^{t} a^{s} c^{t} b a^{s-1} \\
& \text { 14: } b^{t} c^{t} b^{t} a^{t} b^{t} c^{t} a^{t} b^{t} a^{t} c^{t} a^{t} b^{t} c^{t} b^{t-1} a \\
& \text { 17: } c^{t} a^{t} b^{t} c^{t} a^{t} c^{t} b^{t} a^{t} b^{t} c^{t} b^{t} a^{t} c^{t} a^{t} b^{t} c^{t} a b^{t-1}
\end{aligned}
$$

This last claim can easily be verified by hand after noting that $s, t>1$. This concludes the proof.

We now show that almost all binary words of length $n$ are $\beta$-images.

Proof of the main Theorem 1. Let $u \in\{a, b\}^{*}$ be a nonempty binary word of length $n$. We proceed by a case distinction on the number $k_{a}$ of occurrences of the letter $a$ in $u$. Note that $\beta\left(a^{n}\right)=b^{n}$ for the case $k_{a}=0$ and the case $k_{a}=1$ does not exist; see Lemma 1 .

Suppose $k_{a} \geq 2$. If $k_{a} \notin \mathbf{C}$ then there exists a cyclically square-free word $w$ in $A^{*}$ of length $k_{a}$ by Theorem 2, and Lemma 3 shows how to construct a word $w^{\prime}$ such that $\beta\left(w^{\prime}\right)=u$. 
In the remaining case, where $k_{a} \in \mathbf{C}$, we have $a^{n} \notin \beta\left(A^{n}\right)$ which explains the value of $m$; otherwise a cyclically square-free word of length $n \in \mathbf{C}$ would contradict Theorem 2 . Lemma 4 shows that $u$ is a $\beta$-image in the remaining cases.

Finally, by counting, we obtain the number of $\beta$-images: $B_{3}^{n}=2^{n}-n-m$, where $m=1$ if $n \in \mathbf{C}$ and $m=0$ otherwise.

\section{The case of four and more letters}

The exceptions in the Currie set disappear when the alphabet has at least four letters.

Theorem 3. $B_{k}^{n}=2^{n}-n$ for all $k>3$ and $n \geq 2$.

Proof. It is sufficient to prove the claim for the alphabet of four letters, $A=\{a, b, c, d\}$, since $B_{4}^{n}=2^{n}-n$ implies $B_{k}^{n}=2^{n}-n$ for all $k>3$. The $n$ exceptions are the binary words of length $n$ with only one letter $a$; see Lemma 1 . We show that any binary word $u$ of length $n$, except $a b^{n-1}$ and its conjugates, is the $\beta$-image of a word over $A$. Note that $\beta\left(a^{n}\right)=b^{n}$. Let then $u \notin\left[a b^{n-1}\right]$, and suppose $u$ has $k_{a}=m \geq 2$ occurrences of $a$. Let $w$ be the prefix of the square-free Thue word $T$ of length $m$ where the last letter is replaced by $d$, that is, $w=v d$, where $v$ is the prefix of $T$ of length $m-1$. Note that $w$ is cyclically square-free because no square occurs in the prefix $v$, and no square can contain the letter $d$, since $d$ occurs only once in $u$. Now, Lemma 3 implies the claim.

\section{Acknowledgement}

We are grateful to the anonymous referee of this journal for pointing out the second exception of the case $n=10$ in the proof of Lemma 4 .

\section{References}

[1] F. Blanchet-Sadri, E. Clader, and O. Simpson. Border correlations of partial words. Theory Comput. Syst. to appear.

[2] J. D. Currie. There are ternary circular square-free words of length $n$ for $n \geq 18$. Electron. J. Combin., 9(1):Note 10, 7 pp. (electronic), 2002.

[3] L. J. Guibas and A. Odlyzko. String overlaps, pattern matching, and nontransitive games. J. Combin. Theory Ser. A, 30(2):183-203, 1981.

[4] T. Harju and D. Nowotka. Border correlation of binary words. J. Combin. Theory Ser. A, 108(2):331-341, 2004.

[5] M. Lothaire. Combinatorics on Words, volume 17 of Encyclopedia of Mathematics. Addison-Wesley, Reading, MA, 1983.

[6] M. Lothaire. Algebraic Combinatorics on Words, volume 90 of Encyclopedia of Mathematics and its Applications. Cambridge University Press, Cambridge, United Kingdom, 2002. 
[7] D. Moore, W. F. Smyth, and D. Miller. Counting distinct strings. Algorithmica, 23(1):1-13, 1999.

[8] H. Morita, A. J. van Wijngaarden, and A. J. Han Vinck. On the construction of maximal prefix-synchronized codes. IEEE Trans. Inform. Theory, 42:2158-2166, 1996.

[9] A. Thue. Über unendliche Zeichenreihen. Det Kongelige Norske Videnskabersselskabs Skrifter, I Mat.-nat. Kl. Christiania, 7:1-22, 1906.

[10] A. Thue. Über die gegenseitige Lage gleicher Teile gewisser Zeichenreihen. Det Kongelige Norske Videnskabersselskabs Skrifter, I Mat.-nat. Kl. Christiania, 1:1-67, 1912. 